\title{
commentary
}

\section{Reconciling warming trends}

\author{
Gavin A. Schmidt, Drew T. Shindell and Kostas Tsigaridis
}

\begin{abstract}
Climate models projected stronger warming over the past 15 years than has been seen in observations. Conspiring factors of errors in volcanic and solar inputs, representations of aerosols, and El Niño evolution, may explain most of the discrepancy.
\end{abstract}

\begin{abstract}
A ny divergence between real-world climate phenomena and prior expectations poses interesting science questions. The case of the apparent slow-down of warming since the record El Niño event in 1997/1998 is no exception. The global mean surface temperature trend was smaller ${ }^{1}$ between 1997 and $2013\left(0.07 \pm 0.08{ }^{\circ} \mathrm{C}\right.$ per decade $)$ than over the last 50 years $\left(0.16 \pm 0.02{ }^{\circ} \mathrm{C}\right.$ per decade), highlighting questions about the mechanisms that regulate decadal variability in the Earth's temperature. In addition, the warming trend in the most recent 15 -year period is near the lower edge of the $5-95 \%$ range of projections from a collection of climate models that were part of the Coupled Model Intercomparison Project Phase 5 (CMIP5). Why most of the model simulations suggest more warming than has been observed is a second question that deserves further exploration.

Short-term fluctuations in global mean surface temperature anomalies have been
\end{abstract}

a perennial focus of public discussions related to climate change. We should expect to see climate changes, by definition, only in the long-term trends that average over stochastic weather and year-to-year fluctuations such as those associated with the El Niño/Southern Oscillation (ENSO), which favoured a cool La Niña phase in the past few years. On decadal timescales, global mean surface temperatures are expected to vary, too. One cause might be the chaotic internal variability of the coupled system of oceans and atmosphere, for example in the tropical Pacific Ocean ${ }^{2}$, or in variability in deep ocean circulation ${ }^{3}$. Alternatively, decadal-scale temperature variations can be a response of the climate system to external influences, such as volcanic eruptions or the solar cycle. Both kinds of influence will continue to operate and be superimposed on any long-term warming trend from rising atmospheric greenhouse gas concentrations. For shorter time periods - and a 15-year interval may just about qualify as such — natural and externally driven fluctuations can, in principle, lead to a change in warming trend. But it is not obvious that either effect should lead to a discrepancy between climate model projections and observations.

\section{A combination of factors, by coincidence, conspired to dampen warming trends in the real world after about 1992.}

Here we argue that a combination of factors, by coincidence, conspired to dampen warming trends in the real world after about 1992. CMIP5 model simulations were based on historical estimates of external influences on the climate only to 2000 or 2005 , and used scenarios (Representative Concentration Pathways, or RCPs) thereafter ${ }^{4}$. Any recent improvements in these estimates or updates to the present day were not

\section{Box 1 | Adjustments to the CMIP5 external influences}

Well-mixed greenhouse gases. We linearly interpolated observations of atmospheric concentrations of $\mathrm{CO}_{2}, \mathrm{~N}_{2} \mathrm{O}$, $\mathrm{CH}_{4}$ and chlorofluorocarbons (CFCs) between 2005 and 2012, and calculated the radiative effect of these greenhouse gas levels using standard simplified formulae. Concentrations of $\mathrm{CO}_{2}, \mathrm{~N}_{2} \mathrm{O}$ and $\mathrm{CH}_{4}$ were very slightly lower than used in the CMIP5 runs, whereas CFCs were a little higher, leading to a small cool bias in the models (Fig. 1a).

Solar irradiance. Because solar irradiance at the last solar-cycle minimum was lower and the present weak solar cycle started later ${ }^{12}$ than had been assumed in the CMIP5 simulations ${ }^{13}$, solar drivers were too positive in the CMIP5 runs. We multiplied the difference in total solar irradiance forcing by an estimated factor of 2, based on a preliminary analysis of solar-only transient simulations, to account for the increased response over a basic energy balance calculation when whole-atmosphere chemistry mechanisms are included.

Human-made tropospheric aerosols. Only two CMIP5 models include nitrate aerosol forcing, and half did not include aerosol indirect effects. Given the recent increases in Asian sources, nitrate effects amount to perhaps $-0.04 \mathrm{~W} \mathrm{~m}^{-2}$ of additional forcing ${ }^{14}$. Exclusion of aerosol indirect effects ${ }^{15}$ might add another $-0.06 \mathrm{~W} \mathrm{~m}^{-2}$ globally since 2000 . We assumed that this forcing increased slowly in the 1990s, and faster in the 2000s following Asian $\mathrm{NO}_{2}$ emissions ${ }^{16}$, peaking at $-0.1 \mathrm{~W} \mathrm{~m}^{-2}$ in
2010. Underestimates of secondary organic aerosols are an additional missing factor, but are harder to quantify ${ }^{17}$.

Volcanic stratospheric aerosols. A reanalysis of satellite retrievals in the 1990s and additional observations in the 2000 s has produced a record of aerosol optical depth ${ }^{9}$ (updated to version 2.0; ref. 10) that differs significantly from that used in CMIP5 (ref. 18). In particular, the magnitude of the impact of Pinatubo in the early 1990s is downgraded, and larger contributions are suggested for the twenty-first century. Radiative forcing is calculated by assuming no post-2000 volcanic aerosols in CMIP5 and multiplying the difference in aerosol optical depth by -25 to derive a forcing in $\mathrm{W} \mathrm{m}^{-2}$. 
taken into account in these simulations. Specifically, the influence of volcanic eruptions, aerosols in the atmosphere and solar activity all took unexpected turns over the 2000s. The climate model simulations, effectively, were run with the assumption that conditions were broadly going to continue along established trajectories. Additionally, the exact phasing of any natural fluctuations will not be captured in any specific model.

\section{Updated estimates of climate drivers}

To test this hypothesis, we have collated up-to-date information on volcanic aerosol concentrations, solar activity and wellmixed greenhouse gases in the 1990s and 2000s (Box 1). These updates include both newly observed data and also reanalyses of earlier 1990s data on volcanic aerosols based on improved satellite retrievals. Comparing the updated information with the data used in the CMIP5 climate model simulations (Fig. 1a) suggests that in the mid-1990s, cooling factors were overestimated (largely an effect of an overestimate of the stratospheric aerosol optical depth in the wake of the Mount Pinatubo eruption), making the models cooler than the real world. Conversely, in the 2000s, multiple cooling factors were underestimated, so the real world would be expected to have been cooler than in the models.

We estimate how simulated global mean surface temperature would have been different in the CMIP5 runs if two effects had been included: first, if ENSO in each model had been in phase with observations, and second, if the ensemble mean were adjusted using results from a simple impulse-response model $^{5}$ with our updated information on external drivers (Fig. 1b). We find that this procedure reduces the differences between observed and simulated temperatures by a factor of 3 . We conclude that the coincident underestimates of cooling factors in the 2000 s - that is, of volcanic aerosols, solar irradiance and effects of humanmade aerosols - have combined to bias the model ensemble. According to this estimate, about a third of the difference between simulated and observed trends (in the GISTEMP analysis ${ }^{6}$ ) between 1997 to 2013 is a result of underestimated volcanic emissions; about one-seventh of the discrepancy comes from overestimates of solar activity and differences in ENSO phasing between climate models and the real world; and just under a quarter could be related to human-made aerosols. These estimates leave only a small residual between models and observations that

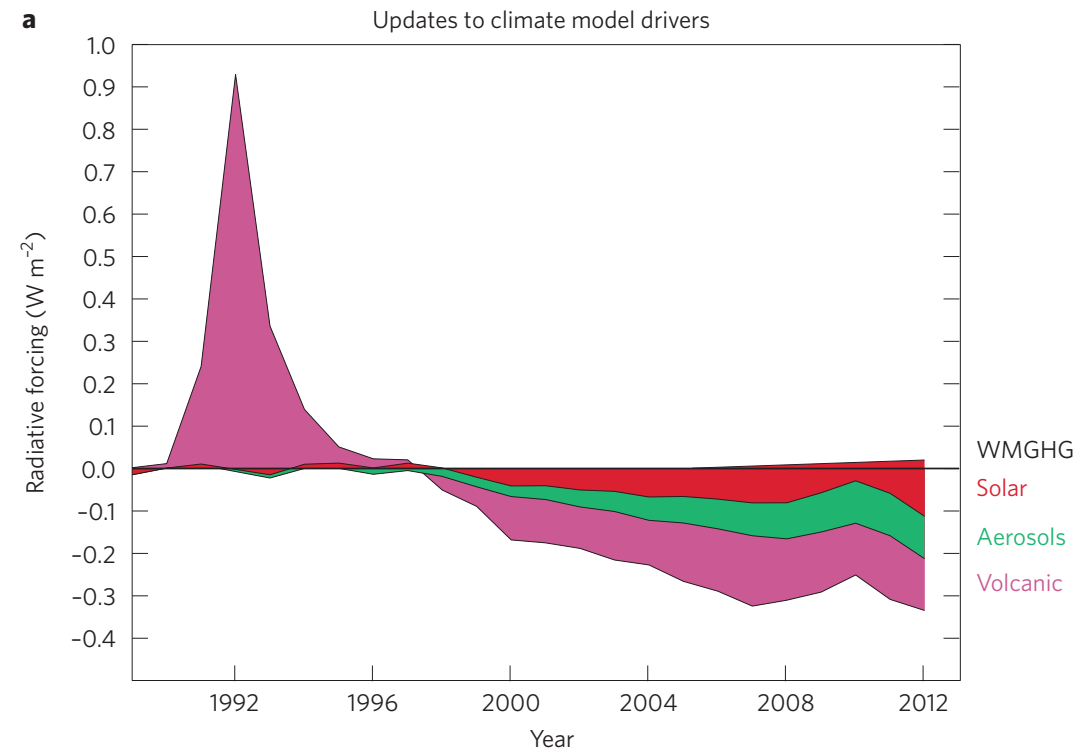

b Global mean temperature anomalies

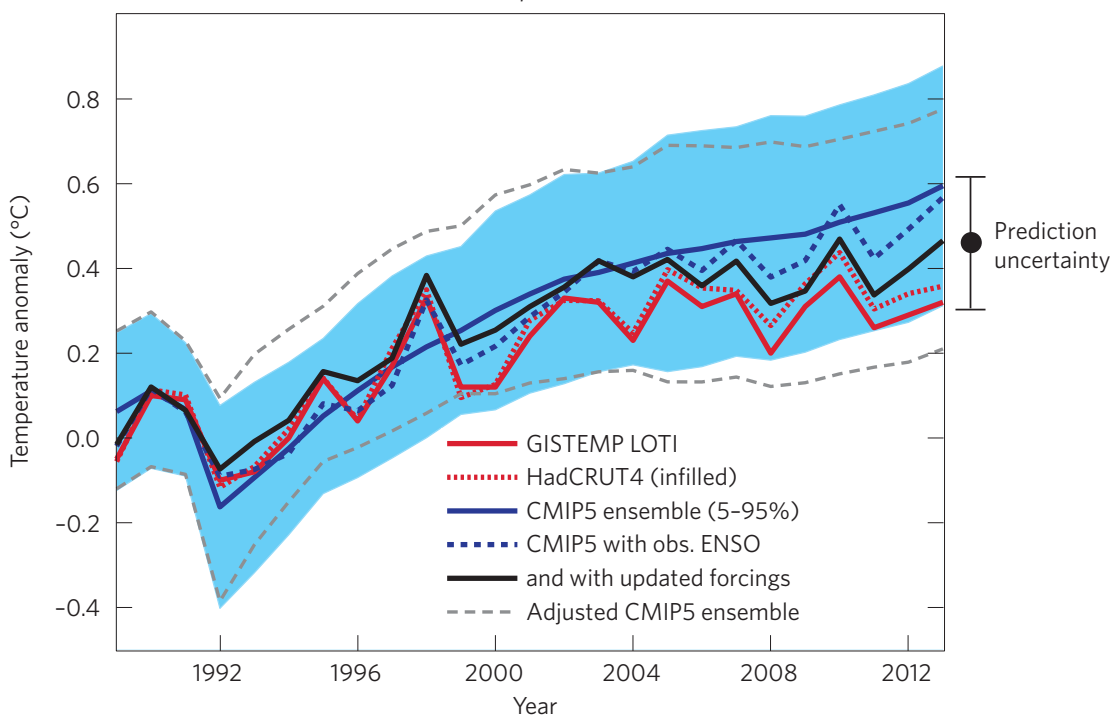

Figure 1 | Updated external influences on climate and their impact on the CMIP5 model runs. a, The latest reconstructions of optical depth for volcanic aerosols $s^{9,10}$ from the Mount Pinatubo eruption in 1991 suggest that the cooling effect of the eruption (1991-1993) was overestimated in the CMIP5 runs, making the simulated temperatures too cool. From about 1998 onwards, however, the cooling effects of solar activity (red), human-made tropospheric aerosols (green) and volcanic eruptions (pink) were all underestimated. WMGHG, well-mixed greenhouse gases. b, Global mean surface temperature anomalies, with respect to 1980-1999, in the CMIP5 ensemble (mean: solid blue line; pale blue shading: 5-95\% spread of simulations) on average exceeded two independent reconstructions from observations (GISTEMP Land-Ocean Temperature Index (LOTI) ${ }^{6}$, solid red; HadCRUT4 with spatial infilling7, dashed red) from about 1998. Adjusting for the phase of ENSO by regressing the observed temperature against the ENSO index ${ }^{11}$ adds interannual variability to the CMIP5 ensemble mean (dashed blue), and adjusting for updated external influences as in a further reduces the discrepancy between model and data from 1998 (black). The adjusted ensemble spread (dashed grey) clearly shows the decadal impact of the updated drivers. As an aside, we note that although it is convenient to use the CMIP5 ensemble to assess expected spreads in possible trends, the ensemble is not a true probabilistic sample.

can be easily accounted for by internal variability unrelated to ENSO, or to any further misspecifications of external influences. In comparison to an alternative temperature analysis ${ }^{7}$ the observed trend matches the adjusted simulated temperature increase even more closely (Fig. 1b). 


\section{Reconciliation}

We conclude that use of the latest information on external influences on the climate system and adjusting for internal variability associated with ENSO can almost completely reconcile the trends in global mean surface temperature in CMIP5 models and observations. Nevertheless, attributing climate trends over relatively short periods, such as 10 to 15 years, will always be problematic, and it is inherently unsatisfying to find model-data agreement only with the benefit of hindsight. We see no indication, however, that transient climate response is systematically overestimated in the CMIP5 climate models as has been speculated ${ }^{8}$, or that decadal variability across the ensemble of models is systematically underestimated, although at least some individual models probably fall short in this respect.
Most importantly, our analysis implies that significant warming trends are likely to resume, because the dominant long-term warming effect of well-mixed greenhouse gases continues to rise. Asian pollution levels are likely to stabilize and perhaps decrease, although lower solar activity may persist and volcanic eruptions are unpredictable. ENSO will eventually move back into a positive phase and the simultaneous coincidence of multiple cooling effects will cease. Further warming is very likely to be the result.

\section{Gavin A. Schmidt, Drew T. Shindell and}

Kostas Tsigardidis are at the NASA Goddard Institute for Space Studies, 2880 Broadway, New York, New York 10025, USA. Kostas Tsigaridis is also at the Center for Climate Systems Research, Columbia University, 2880 Broadway, New York, New York 10025, USA. e-mail: gavin.a.schmidt@nasa.gov
References

1. Fyfe, J. C., Gillett, N. P. \& Zwiers, F. W. Nature Clim. Change 3, 767-769 (2013)

2. Kosaka, Y. \& Xie, S-P. Nature 501, 403-407 (2013).

3. Trenberth, K. E. \& Fasullo, J. T. Earth's Future 1, 19-32 (2013).

4. Taylor, K. E., Stouffer, R. \& Meehl, G. Bull. Am. Meteorol. Soc. 93, 485-498 (2012).

5. Boucher, O. \& Reddy, M. S. Energy Policy 36, 193-200 (2008).

6. Hansen, J., Ruedy, R., Sato, M. \& Lo, K. Rev. Geophys. 48, RG4004 (2010)

7. Cowtan, K. \& Way, R. G. Q. J. R. Meteorol. Soc. http://dx.doi.org/10.1002/qj.2297 (2013).

8. Otto, A. et al. Nature Geosci. 6, 415-416 (2013)

9. Vernier, J-P. et al. Geophys. Res. Lett. 38, L12807 (2011)

10. Santer, B. D. et al. Nature Geosci. 7, 185-189 (2014).

11. Wolter, K. \& Timlin, M. S. Weather 53, 315-324 (1998).

12. Fröhlich, C. \& Lean, J. Geophys. Res. Lett. 25, 4377-4380 (1998)

13. Lean, J. Calculations of Solar Irradiance (2009); http://go.nature.com/VbldYY

14. Shindell, D. T. et al. Atmos. Chem. Phys. 13, 2939-2974 (2013) 15. Bellouin, N. et al. J. Geophys. Res. 116, D20206 (2011).

16. Ma, J. Z. et al. Atmos. Chem. Phys. 13, 1547-1567 (2013).

17. Hoyle, C. R. et al. Atmos. Chem. Phys. 11, 321-343 (2011)

18. Sato, M., Hansen, J. E., McCormick, M. P. \& Pollack, J. B. J. Geophys. Res. 98, 22987-22994 (1993).

\title{
Bumpy path to a warmer world
}

\author{
Martin Visbeck
}

\section{Decadal climate variability has long received limited attention. With the slow-down in surface warming since the late 1990s, the decadal scale has rightly become a focus of attention: for assessing climate change and its impacts, it is of critical importance.}

A changing climate matters most to our generation on timescales from seasons to decades. Decadal climate information, in particular, is essential for societies in developing to become climate-resilient.

The developed world has established a wealth of adaptation mechanisms to cope with the effects of a changing climate on seasonal and interannual timescales. Advanced water management systems, capital reserves and widespread insurance all help to buffer the loss, damage and gains from short-term climate fluctuations. By contrast, a 10-year drought, or a decade of high tropical storm activity, may easily exhaust the coping strategies of even the most developed economies. In the developing world, coping mechanisms are less abundant, and the same change in the climate often leads to great societal hardship.

Beyond the decadal timescale, on the other hand, the world is developing longterm adaptation strategies to cope with persistent climate change. These include the development of large-scale infrastructure

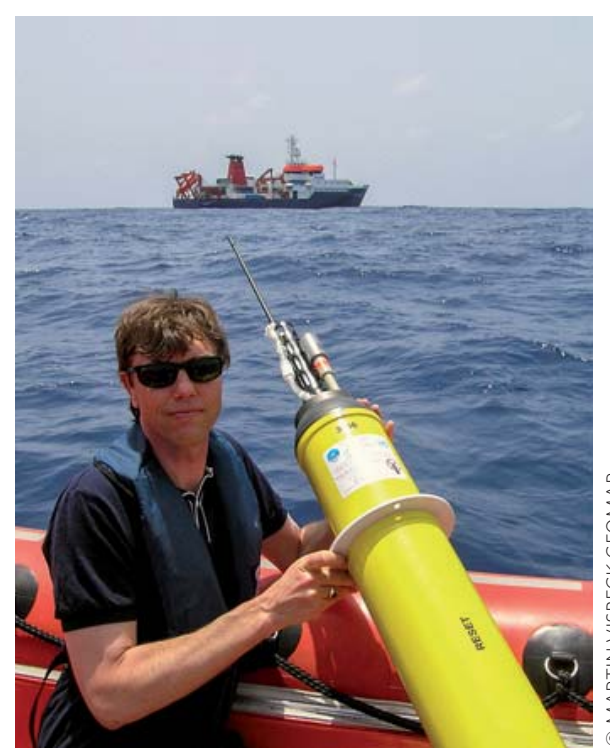

Figure 1 | Ocean observations. Martin Visbeck deploying an Argo float in the ocean from the research vessel MV Maria S. Merian. Argo is an international network of more than 3,000 robotic floats that measure the temperature and salinity of the ocean's upper $2,000 \mathrm{~m}$. such as seawalls and water reservoirs, alternative responses such as retreat from affected land, and adjustments in economic and legislative structures such as insurance frameworks.

Climate variability on scales of 10 to 30 years - too long to sit out, yet too short to adapt to fully - is therefore particularly important to our generation. Nevertheless, the slow-down in warming over the past 15 years or so - despite unabated emissions of greenhouse gases and global climate model projections that suggest much steeper warming - has caught parts of the scientific community by surprise. We should have expected such decadal swings if more emphasis had been devoted to understanding the decadal scale.

\section{Weak and regional}

The most energetic frequency range in almost any long-term climate record is the annual cycle. In the tropics, the annual band is followed by climate variability on timescales of 3 to 7 years associated with the El Niño/Southern Oscillation (ENSO), a large-scale climate see-saw that is rooted 